\title{
Primed and unprimed rebounding illusory apparent motion
}

\author{
Nicolas Davidenko ${ }^{1}$ - Nathan H. Heller ${ }^{1}$ \\ Published online: 16 January 2018 \\ (C) The Psychonomic Society, Inc. 2018
}

\begin{abstract}
Although sequences of uncorrelated random dots can yield a wide range of illusorily coherent motion percepts (including translation, rotation, contraction, expansion, shear, and rebounding motion), past priming studies have relied on twoalternative forced choice tasks that only measure unidirectional (positive or negative) priming effects. In Experiment 1 we showed that when participants are primed with unidirectional motion and given an additional option to report bidirectional (rebounding) motion, they do so frequently, suggesting that unidirectional motion can "default" to a rebounding percept. Furthermore, rebounding percepts are more prevalent during trials with long frame durations, suggesting a role for attention in forming and maintaining these illusory percepts. In Experiment 2 we compared rebounding percepts that followed unidirectional, drifting primes with rebounding percepts that followed bidirectional, rebounding primes, and found that these two types of illusory rebounding motion percepts differ systematically in their temporal structures. We argue that rebounding percepts following drifting primes can be understood as a breakdown of positive priming into an underlying oscillatory state, whereas rebounding percepts following rebounding primes may be understood either as (1) the initialization of the same oscillatory process, or (2) the entrainment of a two-step motion pattern by a higher-order mechanism.
\end{abstract}

Keywords Apparent motion · Priming $\cdot$ Adaptation · Aftereffects

Apparent motion (AM) is the perception of coherent motion elicited by the sequential presentation of two or more spatiotemporally correlated images. In recent studies, we have explored a phenomenon we term illusory apparent motion (IAM), in which spatiotemporally uncorrelated images lead to globally coherent percepts of AM. These percepts are illusory in the sense that they are selected from an unconstrained space of possible correspondence solutions. In one of our recent studies (Davidenko, Heller, Cheong, \& Smith, 2017), we showed that priming participants with unidirectional (drifting) apparent motion patterns leads to persistent IAM that continues to drift in the primed direction in subsequent sequences of

Electronic supplementary material The online version of this article (https://doi.org/10.3758/s13414-018-1483-1) contains supplementary material, which is available to authorized users.

Nicolas Davidenko ndaviden@ucsc.edu

1 Department of Psychology, University of California, Santa Cruz, CA, USA uncorrelated random-dot arrays. This phenomenon is closely related to an effect known as "visual inertia," which was first studied by Anstis and Ramachandran (1987) in bistable motion quartets - simple ambiguous apparent motion stimuli that can be perceived as moving either vertically or horizontally. Visual inertia was observed when an unambiguous motion signal (i.e., vertical or horizontal motion) biased the subsequent perception of a bistable motion quartet such that its perceived motion became consistent with the preceding unambiguous motion signal. Anstis and Ramachandran described this effect in terms of competitive interactions between local directionally selective motion detectors that solve the ambiguous correspondence problem. In earlier work, Ramachandran and Anstis (1985) showed that such competitive interactions were able to propagate from a local quartet to a global field of quartets, causing a coherent global motion pattern to emerge. However, for such a mechanism to account for the emergence of drifting IAM in maximally ambiguous sequences of uncorrelated random-dot arrays (Davidenko et al., 2017), the types of local-to-global competitive interactions described by Anstis and Ramachandran would have to be flexible enough to converge on a particular correspondence solution from an unbounded space of possible correspondence solutions. 
Visual inertia, more recently referred to as "positive priming," can be systematically dissociated from the competing phenomenon of "negative priming," which is related to the flicker motion aftereffects studied by Nishida and Sato (1992, 1995). In negative priming, the correspondence problem is solved in the opposite direction from the priming motion, which is believed to result from the adaptation of directionally selective motion detectors. Whether motion priming leads to positive or negative effects has been shown to depend on a variety of stimulus parameters (Heller \& Davidenko, 2018; Kanai \& Verstraten, 2005; Takeuchi, Tuladhar, \& Yoshimoto, 2011; Yoshimoto, Uchida-Ota, \& Takeuchi, 2014), as well as on the deployment of visual attention (Culham, Verstraten, Ashida, \& Cavanagh, 2000). Specifically, longer frame durations, slower stimulus velocities, and shorter prime durations facilitate positive priming effects, whereas shorter frame durations, faster stimulus velocities, and longer prime durations facilitate negative priming effects. Moreover, effortful attentional tracking facilitates positive priming, whereas passive viewing facilitates negative priming. Thus, our finding that unidirectional, drifting motion induces persistent drifting IAM can be accounted for by known mechanisms based on stimulus features, competitive interactions between motion detectors, and the deployment of visual attention.

However, in the same study (Davidenko et al., 2017), we also showed that priming with bidirectional (rebounding) motion patterns that alternate back and forth across successive frames leads to persistent rebounding IAM, in which the illusory motion continues to rebound, as if reinstantiating a primed two-step motion pattern. Although drifting IAM can be understood as an instance of positive priming, rebounding IAM is not explained well by the same motion priming mechanism. What mechanism(s) can account for rebounding IAM?

One possibility is that rebounding IAM relies on a mechanism similar to the one that supports drifting IAM, but operating over a larger temporal window. To support the two-step, bidirectional nature of rebounding motion, such a mechanism would require a higher-level component that encodes multiple events (i.e., two frame transitions), keeps track of their temporal order, and then deploys selective attention to solve the appropriate correspondence problems on subsequent frame transitions. This would constitute a high-level cognitive, memory-based pattern recognition mechanism.

Another possibility is that rebounding IAM arises spontaneously as a "default" motion percept, without requiring such high-level cognitive processes. A number of past studies that have explored the underlying mechanisms for unidirectional and bidirectional motion patterns have suggested different accounts for why rebounding motion might arise as a default percept (Hock, Park, \& Schöner, 2002; Hsieh, Caplovitz, \& Tse, 2005; Verstraten, Cavanagh, \& Labianca, 2000). For instance, a default account of rebounding motion might stem from a prevalence of passive versus active viewing. Using simpler ambiguous apparent motion stimuli, Verstraten et al. described that effortful attentional tracking is required to perceive continuous (viz. drifting) rotational motion, but that passive viewing is sufficient to perceive rocking (viz. rebounding) rotational motion in the same ambiguous stimuli. Similarly, the default nature of rebounding motion might arise due to the prevalence of diffused versus focused spatial attention. Hock et al. showed that narrowly tuned spatial attention facilitates continuous (drifting) motion percepts, whereas broadly tuned spatial attention facilitates oscillating (rebounding) motion percepts. Thus, rebounding IAM might arise as a default percept whenever effortful attention is limited and/or spatial attention is spread. An ecologically plausible explanation for the default perception of rebounding motion may stem from a lifetime of experience with stationary objects that sway, shake, or bounce, without necessarily moving toward a destination (see Hsieh et al., 2005).

If rebounding IAM is a "default" percept, then it should be observed under a wide variety of circumstances. Indeed, Hsieh and Tse (2006) showed in a passive viewing task that sequences of uncorrelated random dots presented on a square array are often reported to rebound back and forth across the cardinal or diagonal axes of the square. Likewise, in our own past studies we have shown that rebounding IAM is the most likely percept to arise spontaneously (without any priming) in the context of a motion detection task (Davidenko, Cheong, \& Smith, 2015). Moreover, the participants in our priming study (Davidenko et al., 2017) anecdotally reported that some percepts that begin as drifting IAM eventually "fall back" to rebounding IAM. Taken together, these findings further support the view that "rebounding" is a default state of the motion-processing system.

Our first experiment here was designed to answer a question left open from a recent study (Heller \& Davidenko, 2018) in which we primed participants with unidirectional rotational apparent motion and tested their subsequent perception of a sequence of uncorrelated random-dot arrays. We found that when participants were given the option to report perceiving "something else" besides just positive or negative priming, they did so in a significant proportion of trials (about $17 \%$ across conditions). Could some proportion of these "something else" responses reflect rebounding percepts? To address this question, in Experiment 1 we tested whether unidirectional (drifting) motion primes lead to bidirectional (rebounding) IAM by running a modified version of Heller and Davidenko's task. In each trial, participants were primed with rotational apparent motion on an annulus-shaped display and asked to report any motion they perceived in a subsequent sequence of uncorrelated random dots. In a four-alternative forced choice (4AFC) task, participants could report drifting clockwise $(\mathrm{CW})$, drifting counterclockwise (CCW), rebounding, or "something else" (SE). Across trials we 
manipulated the frame duration of the stimuli. Given our past findings, we predicted that long frame durations (500 and $1,000 \mathrm{~ms}$ ), in which attention can exert an influence, should primarily produce positive priming, whereas short frame durations $(62.5,125$, and $250 \mathrm{~ms})$ should primarily produce negative priming. If rebounding IAM is a default percept, we should observe reports of rebounding motion in this task despite never priming rebounding motion.

Where Experiment 1 was designed to measure the extent to which unidirectional, drifting motion patterns "default" to illusory rebounding percepts, Experiment 2 was designed to address two goals: (1) to better characterize the temporal structure of the percepts reported as "rebounding" in Experiment 1, and (2) to compare the temporal structures of rebounding percepts that follow drifting versus rebounding motion primes. Thus, in Experiment 2 we elicited CW, CCW, or SE responses on a frame-by-frame basis, while manipulating the type of priming (drifting or rebounding) across blocks.

\section{Experiment 1: Unprimed rebounding percepts}

\section{Method}

Participants The participants were 23 undergraduate students at the University of California, Santa Cruz, who gave informed consent and participated in exchange for course credit. The study was approved by UCSC's Institutional Review Board.

Stimuli Frames for the apparent motion stimuli were constructed and presented using MATLAB. Each frame was composed of a $300 \times 300$ array of random grayscale pixels. The array was blurred using a low-pass filter with a two-pixel radius and masked with an annulus, and a fixation dot was positioned at the center of the annulus (see Fig. 1). The diameters of the outer and inner rings of the annulus spanned $12.0^{\circ}$ and $4.5^{\circ}$ of visual angle, respectively. CW and CCW global motion percepts were induced by superimposing a lowspatial-frequency radial sine-wave grating on each new frame, thus imputing an unambiguous apparent motion signal to the underlying array of randomly changing pixels, via a phenomenon known as motion capture (Ramachandran \& Cavanagh, 1987). The radial sine-wave grating with a $90^{\circ}$ cycle around the annulus was rotated by a constant angle of $22.5^{\circ}$ between successive frames, corresponding to a one-quarter-cycle jump per frame. Across trials, we manipulated the frame duration in the priming and test phases $(62.5,125,250,500$, and 1,000 $\mathrm{ms})$. To keep the total duration of the priming and test phases constant at 5 and $2 \mathrm{~s}$, respectively, we varied the numbers of priming and testing frames across the five frame durations (i.e., $80,40,20,10$, or 5 priming frames and $32,16,8,4$, or 2 testing frames, respectively).
Procedure The contrast of the rotating sine-wave grating decreased by $20 \%$ every second throughout the priming phase, disappearing the moment the test phase began; the test phase consisted only of randomly refreshing arrays of blurred pixels on an annulus mask. The priming phase was differentiated from the test phase by the presence of a black fixation dot during the priming phase that turned white at the onset of the test phase. Participants were instructed to fixate on the black dot while paying attention to the global motion pattern defined by the sine-wave gratings, and then to report what they perceived after the fixation dot became white (i.e., during the test phase). To ensure that participants were following the instructions, catch trials were included in which the sine-wave gratings remained visible at $20 \%$ contrast throughout the test phase (see Video 1 for an example trial and catch trial with 125-ms frames, and Video 2 for an example trial and catch trial with $500-\mathrm{ms}$ frames). There were a total of 100 noncatch trials ( 5 frame durations $\times 2$ directions $\times 10$ repetitions) and 20 catch trials, resulting in a 15-min experiment.

\section{Results}

$\mathrm{CW}$ and $\mathrm{CCW}$ responses were classified as "positive" or "negative" priming depending on whether they matched or mismatched, respectively, the direction of the priming motion. Figure 2 shows the proportions of trials on which positive priming (solid black), negative priming (solid gray), rebounding (dotted black), and SE (dotted gray) were reported as a function of frame duration. The solid lines show a systematic increase in the proportion of positive priming at longer frame durations (black), with a complementary decrease in the proportion of negative priming (gray). This pattern replicates our previous findings dissociating positive from negative priming effects by manipulating frame duration (see Heller \& Davidenko, 2018).

The dotted black line, representing the proportion of rebounding responses, clearly demonstrates that participants do report perceiving rebounding IAM (on an average of $17.3 \%$ of trials across conditions), even though they are only ever primed with unidirectional motion. Intriguingly, the proportion of rebounding responses is not constant across conditions, but depends on the frame duration [one-way repeated measures analysis of variance (ANOVA): $F(4,88)=4.22, p=.004]$. Post-hoc $t$ tests showed that the proportions of rebounding responses were significantly higher at long frame durations $(27.0 \%$ at $500 \mathrm{~ms}$ and $21.4 \%$ at $1,000 \mathrm{~ms}$ ) than at short frame durations $(9.9 \%$ at $62.5 \mathrm{~ms}, 12.4 \%$ at $125 \mathrm{~ms}$, and $15.9 \%$ at $250 \mathrm{~ms}), t(22)=2.24, p$ $=.04$. Thus, although drifting motion primes often "default" to rebounding illusory percepts, the chance of this happening is not equally likely across all frame durations.

Although the results of Experiment 1 are suggestive that rebounding motion arises as a default percept, the reporting options presented in the 4AFC task required participants to summarize their percept during the entire test phase (i.e., a 


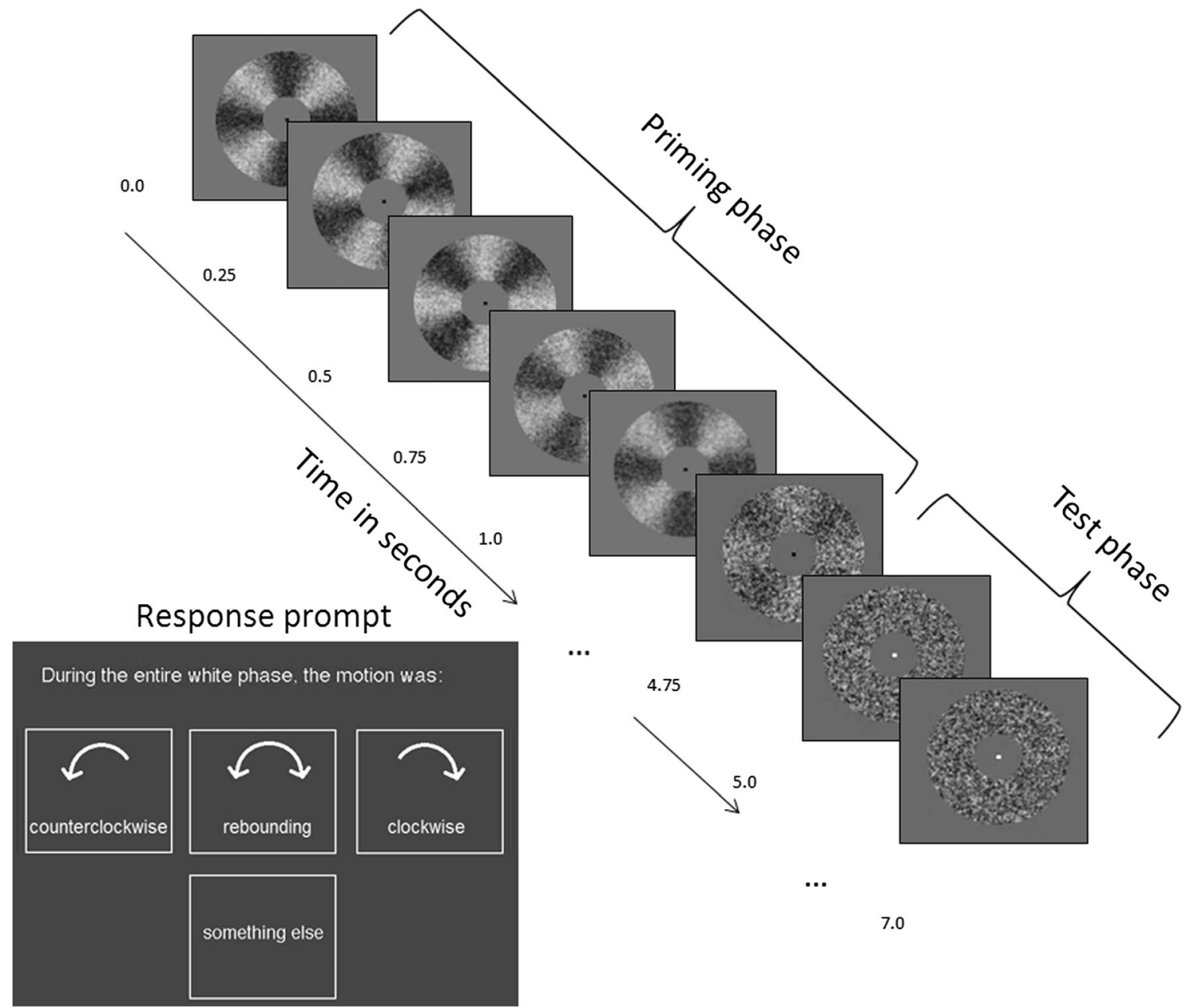

Fig. 1 Schematic of the experimental stimuli and procedure for Experiment 1. Arrays of randomly refreshing dots superimposed on rotating sine gratings produce a priming motion pattern. Participants

motion pattern. They were then asked to report the direction of the motion perceived during the test phase, after the sine grating had faded away, by clicking on one of the four response options shown at the lower left were instructed to fixate on the central dot while attending to the global

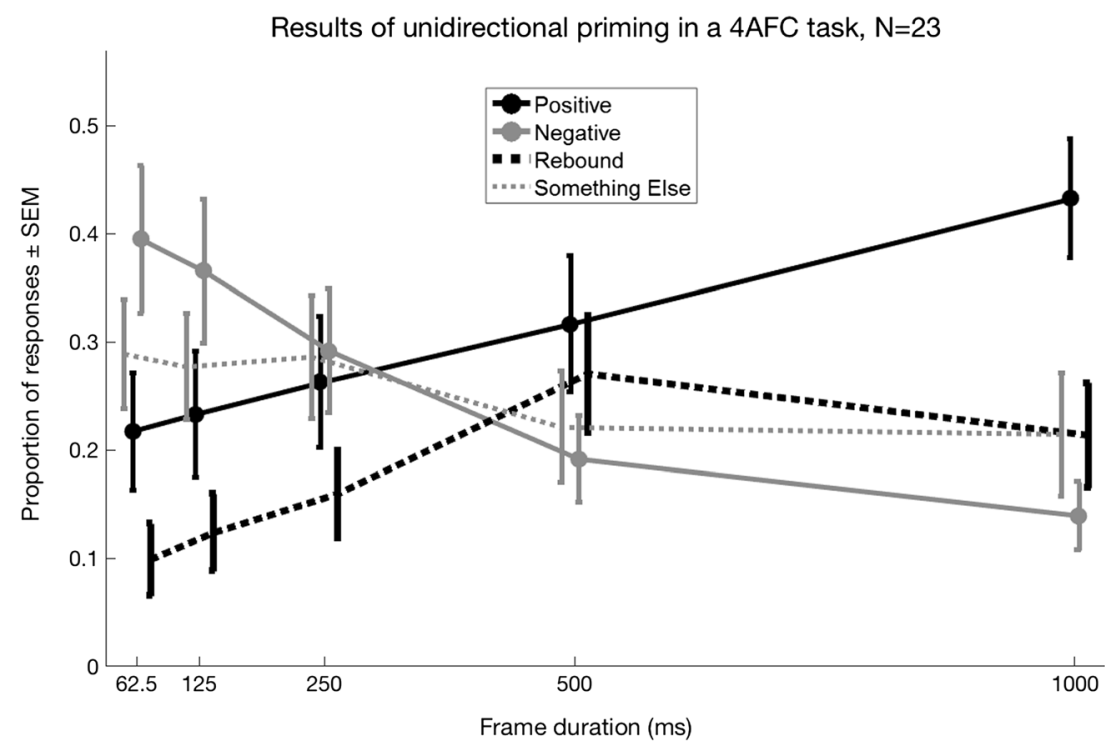

Fig. 2 Average proportions of responses across priming conditions: Positive priming (solid black), negative priming (solid gray), rebounding (dotted black), and "something else" (SE; dotted gray) are plotted against frame duration. The frame durations were always matched between the priming phase and the test phase. Error bars denote standard errors of the means across subjects 
2-, 4-, 8-, 16-, or 32-frame period ) with a single category label. This leaves open the possibility that the "rebounding" reports may have reflected different percepts across observers and across conditions. For example, some observers may have classified an illusory pattern of the form $\mathrm{CW}-\mathrm{CW}-\mathrm{CCW}-$ $\mathrm{CCW}$ as rebounding, even though the pattern did not strictly alternate back and forth from frame to frame. To address this caveat, in Experiment 2 we reduced the test period to just two frames (keeping the frame duration fixed at $500 \mathrm{~ms}$ ) and asked participants to report with two button presses the sequence of motion steps perceived during the two-frame test period. This two-step reporting paradigm allowed participants to characterize their experienced percepts more explicitly, without relying on participants' interpretations of "rebounding." In addition, this paradigm allowed us to distinguish between two types of rebounding percepts: a positive-negative rebound (Pos/Neg Rebound) pattern that begins with a "positive" illusory motion step (following the same direction as the last priming frame) followed by a "negative" illusory motion step (opposite to the last priming frame). In contrast, a negative-positive rebound (Neg/Pos Rebound) pattern would begin with a negative step and continue with a positive step.

Furthermore, to differentiate between rebounding as a "default" percept versus rebounding as a "primed" percept, Experiment 2 included two separate blocks of trials: a "drifting block," in which the priming motion was always unidirectional (e.g., $\mathrm{CW}$ or $\mathrm{CCW}$ throughout the priming phase), and a "rebounding block," in which the priming motion was always bidirectional (i.e., alternating between $\mathrm{CW}$ and $\mathrm{CCW}$ across successive frames). On the basis of past studies in which we primed both drifting and rebounding IAM (Davidenko et al., 2017), we made two predictions. First, rebounding percepts should be prevalent during drifting blocks (as in Exp. 1), but even more prevalent during rebounding blocks. Second, the relative proportion of Pos/ Neg Rebounding versus Neg/Pos Rebounding percepts should differ systematically across blocks. If rebounding motion can be primed as a two-step motion pattern, then the first illusory frame following a rebounding prime will be more likely to go in the opposite direction from the final priming frame (e.g., priming rebounding motion that ends with $\mathrm{CW}-$ $\mathrm{CCW}$ should lead primarily to rebounding percepts that begin with CW illusory motion).

\section{Experiment 2: Comparing unprimed and primed rebounding percepts}

\section{Method}

Participants The participants were 45 undergraduate students at the University of California, Santa Cruz, who gave informed consent and participated in exchange for course credit.
The study was approved by UCSC's Institutional Review Board.

Stimuli The stimuli were similar to those in the 500-ms condition of Experiment 1, with the following changes: (a) the random-dot array was based on a $250 \times 250$ pixel texture (as opposed to $300 \times 300$ in Exp. 1); (b) each trial consisted of nine priming frames and two test frames; and (c) across blocks, we manipulated the motion pattern of the priming stimulus between unidirectional drifting primes (as in Exp. 1; see Video 3 for an example trial) and bidirectional rebounding primes (in which the priming motion itself alternated back and forth across frames; see Video 4). The order of blocks was counterbalanced across participants, with 22 participants starting with the drifting block and 23 participants starting with the rebounding block.

Procedure After each trial, participants were prompted to press two buttons sequentially to describe the motion $(\mathrm{CW}$, $\mathrm{CCW}$, or SE) in each of the two test frames. This created a total of nine possible response combinations, which we classify in the analysis as is shown in Table 1 (assuming a CW final priming frame).

To confirm that this task was feasible, we trained participants to respond to just two test frames (with no priming) that showed all combinations of $\mathrm{CW}$ and/or CCW motion (see Video 5 for examples of the training trials). Participants completed sets of 12 training trials until their performance reached $70 \%$ accuracy. Most participants found this task easy, and 43 of 45 achieved $70 \%$ accuracy after just one set of training trials (one participant required two sets of training trials to pass, and one participant failed after six sets of training trials and was not included in the analysis). In addition, $20 \%$ of the trials were "catch trials" with veridical motion in the two test

Table 1 Nine possible response combinations in Experiment 2, classified into Positive, Negative, Pos/Neg Rebounding, Neg/Pos Rebounding, or Something Else (SE) percepts

\begin{tabular}{lll}
\hline Response 1 & Response 2 & Classification \\
\hline $\mathrm{CW}$ & $\mathrm{CW}$ & Positive priming \\
$\mathrm{CCW}$ & $\mathrm{CCW}$ & Negative priming \\
$\mathrm{CW}$ & $\mathrm{CCW}$ & Pos/Neg rebounding \\
$\mathrm{CCW}$ & $\mathrm{CW}$ & Neg/Pos rebounding \\
$\mathrm{CW}$ & $\mathrm{SE}$ & Something else \\
$\mathrm{CCW}$ & $\mathrm{SE}$ & Something else \\
$\mathrm{SE}$ & $\mathrm{CW}$ & Something else \\
$\mathrm{SE}$ & $\mathrm{CCW}$ & Something else \\
$\mathrm{SE}$ & $\mathrm{SE}$ & Something else \\
\hline
\end{tabular}

These classifications are defined relative to the motion direction in the final priming frame (in this case, clockwise [CW] motion). Analogous classifications were defined relative to a counterclockwise $(\mathrm{CCW})$ final priming frame for those trials. 
frames, similar to the training trials shown in Video 5. In half of these catch trials, the motion-carrying sine grating was high-contrast (easy catch trials), and in the other half it was low-contrast (hard catch trials). Overall, the mean performance on these catch trials was $69.5 \%$ and $60.0 \%$, respectively, indicating that participants could perform the task well above chance $(11.1 \%)$. Below we present the results from the 44 participants who passed the training phase, regardless of their performance on the catch trials, since our results did not differ for high- versus low-performers.

\section{Results}

The results from the drifting-block trials are shown in Fig. 3A. Overall, priming with unidirectional drifting motion at $2 \mathrm{~Hz}$ resulted in a plurality of positive responses on the two test frames (i.e., $\mathrm{CW}-\mathrm{CW}$ if the priming was $\mathrm{CW}$, and $\mathrm{CCW}-$ $\mathrm{CCW}$ if the priming was $\mathrm{CCW}$ ), which accounted for $42.5 \%$ of all responses. In contrast, the prevalence of two "negative" responses was only $7.2 \%$, suggesting that our new paradigm led predominantly to positive priming. As expected, a large number of response pairs reflected "rebounding" percepts, with $21.1 \% \mathrm{Neg} / \mathrm{Pos}$ Rebounding and $11.8 \% \mathrm{Neg} / \mathrm{Pos}$ Rebounding. Taken together, rebounding responses made up $32.9 \%$ of all responses, replicating our findings from Experiment 1 that priming with unidirectional motion at long frame durations leads to frequent reports of rebounding IAM. Similarly, $17.4 \%$ of the response pairs were classified as "Something Else" (with at least one button press being SE), again replicating our previous findings.

The results from the rebounding-block trials (Fig. 3B) showed that when rebounding motion is primed, an even greater proportion of responses during the test phase reflect rebounding percepts. In total, rebounding percepts accounted for $50.5 \%$ of the responses in trials with rebounding primes, whereas positive $(17.2 \%)$ and negative $(18.8 \%)$ only accounted for a combined $35.9 \%$ of the responses, and SE responses were reported on $13.6 \%$ of trials. These results support our claim that rebounding motion is even more likely to arise following rebounding primes than following drifting primes [paired $t$ test: $t(43)=3.47, p=.001$ ]

Intriguingly, the rebounding percepts following drifting and rebounding primes differed systematically in their temporal structure. Specifically, the majority of rebounding responses following drifting primes (64\%) were of the type $\mathrm{Pos} / \mathrm{Neg}$, whereas the majority of rebounding responses following rebounding primes (73\%) were of the type Neg/Pos. A paired $t$ test shows that this interaction was highly significant and consistent across participants $[t(43)=7.4, p=$ .000000003 ; see Fig. 4]. The systematic difference in the prevalences of Pos/Neg Rebounding versus Neg/Pos Rebounding across the two types of primes is suggestive of separate

a

Drifting block
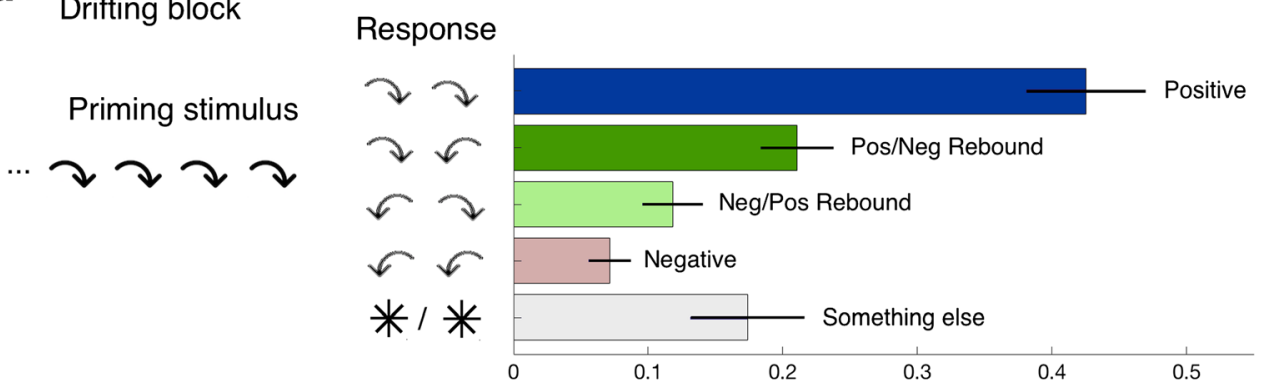

b Rebounding block

Response
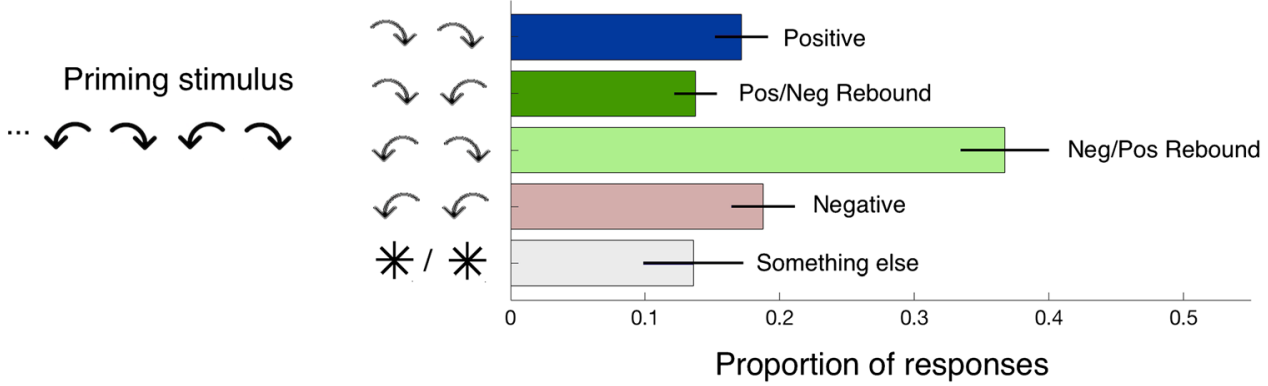

Fig. 3 Distributions of responses to random frames following priming with drifting (A) or rebounding (B) motion. (A) Data from the drifting block are collapsed across clockwise (shown) and counterclockwise (not shown) primes. The pairs of responses are coded as Positive, Pos/Neg Rebound, Neg/Pos Rebound, Negative, or Something Else relative to the

final priming frame. (B) Data from the rebounding block are collapsed across final priming frames moving clockwise (shown) and counterclockwise (not shown). Error bars denote one standard error of the mean across 44 participants 


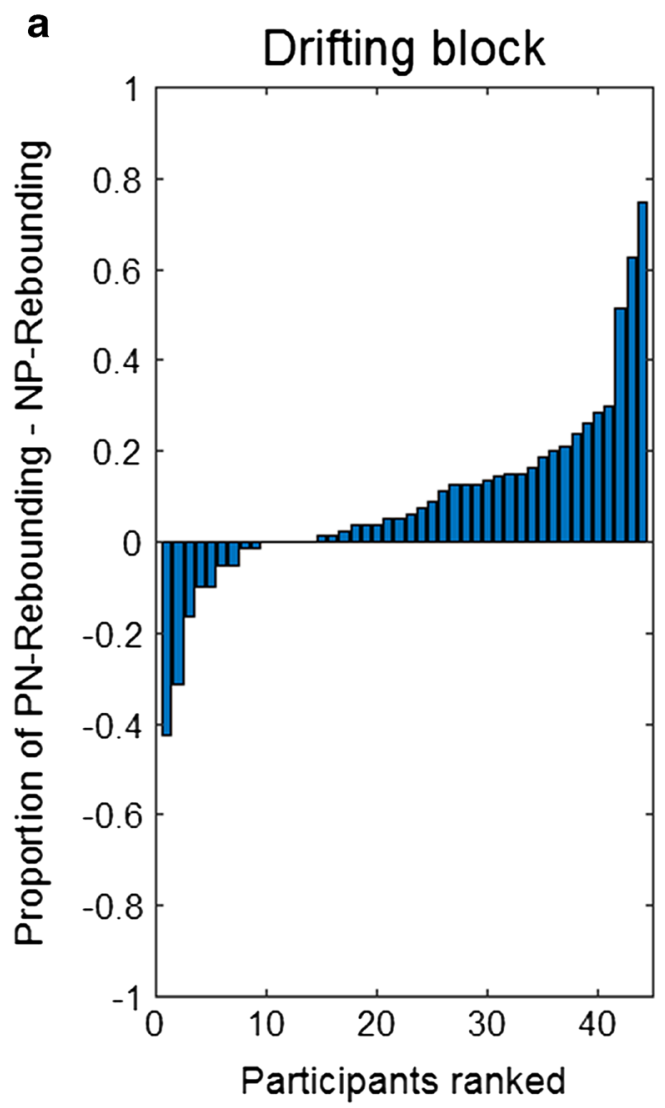

Fig. 4 Proportions of Pos/Neg Rebounding minus Neg/Pos Rebounding responses, shown for each participant during drifting trials (A) and rebounding trials (B) and ranked from the smallest to the greatest difference. (A) During drifting trials, 30 out of 44 participants (68\%) showed a greater proportion of Pos/Neg than of Neg/Pos responses, nine showed

mechanisms. In particular, the prevalence of Neg/Pos Rebounding percepts following rebounding primes may indicate the encoding and reinstatement of an alternating motion sequence, a type of pattern completion phenomenon that has been previously observed in the perception of ambiguous apparent-motion quartets (see Maloney, Dal Martello, Sahm, \& Spillmann, 2005).

\section{Discussion}

Our data from Experiment 1 replicated our previous findings (Heller \& Davidenko, 2018) showing that positive and negative priming can be elicited in maximally ambiguous sequences of uncorrelated random-dot arrays and can be dissociated from each other by manipulating the frame duration of the priming and test stimuli. Short frame durations predominantly lead to negative priming, whereas long frame durations predominantly lead to positive priming. Furthermore, the results of this study yielded two novel findings: (1) Participants often report perceiving rebounding IAM despite never having been primed with rebounding motion, and (2) rebounding

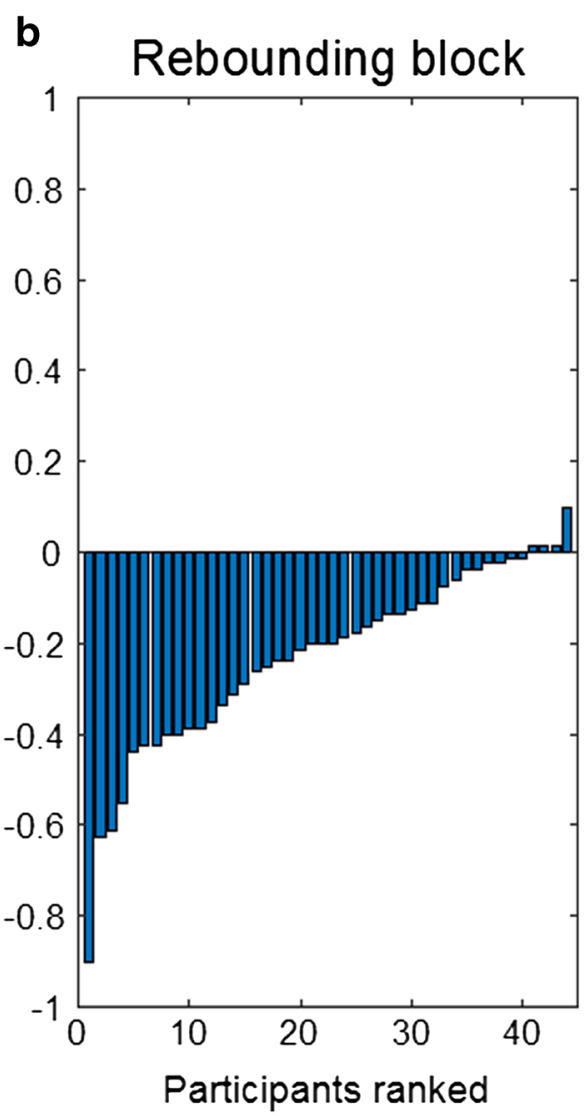

the opposite pattern, and five showed no difference. (B) During rebounding trials, 40 out of 44 participants $(91 \%)$ showed a greater proportion of Neg/Pos than of Pos/Neg responses, and just four showed the opposite pattern

percepts are more prevalent during trials with long frame durations, in which attentional mechanisms are known to play a role (Culham et al., 2000; Heller \& Davidenko, 2018; Kanai \& Verstraten, 2005; Verstraten et al., 2000).

The drifting blocks in Experiment 2 replicated the results of Experiment 1 using a new response paradigm, showing that a substantial number of rebounding percepts are reported following unidirectional primes. The rebounding blocks of Experiment 2 replicated previous findings (Davidenko et al., 2017) in which we showed that priming with a rebounding pattern produces illusory rebounding percepts. Moreover, a comparison of frame-by-frame responses across blocks revealed a systematic difference in the temporal structures of illusory rebounding percepts that followed a drifting prime versus a rebounding prime. Specifically, drifting primes led primarily to Pos/Neg Rebounding percepts, whereas rebounding primes led primarily to Neg/Pos Rebounding percepts. In these Neg/Pos Rebounding responses, the first illusory step matched the directionality of the second-to-last priming frame, and the second illusory step matched the directionality of the final priming frame. As we discuss below, the difference between the predominance of $\mathrm{Pos} / \mathrm{Neg}$ 
Rebounding versus Neg/Pos Rebounding may be indicative of separate mechanisms.

\section{Mechanisms of primed versus unprimed rebounding percepts}

What mechanisms can account for the unprimed rebounding motion percepts that followed unidirectional drifting primes in Experiment 1 and in the drifting blocks of Experiment 2? One plausible mechanism involves rivalry-like effects between directionally selective neural populations. For example, neural populations that are tuned to similar motion directions may be related by excitatory connections that stabilize a globally coherent motion percept. Meanwhile, oppositely tuned neural populations may be related by reciprocal inhibitory connections that induce oscillatory effects, leading to motion reversals. Such a system has been previously proposed to model binocular-rivalry dynamics (Laing \& Chow, 2002; Lehky, 1988; Mueller, 1990). Thus, rebounding motion that follows a drifting prime may be interpreted as short-lived positive priming of a directionally selective neural population that succumbs to the inhibitory influences of opposing neural populations either after one random frame (i.e., Pos/Neg Rebounding, $21.1 \%$ of trials) or immediately after priming (Neg/Pos Rebounding, $11.8 \%$ of trials).

If we can explain the presence of such unprimed rebounding percepts as instances in which positive priming falls back to a default oscillatory state, can the same mechanism explain the pattern of primed rebounding responses following rebounding primes? One possibility is that a rebounding motion prime sets the initial conditions of a default oscillatory state to a specific motion pattern (e.g., CW, $\mathrm{CCW}, \mathrm{CW}, \mathrm{CCW}$ ), and when the rebounding motion prime ends, the subsequent illusory rebounding percept continues in the same temporal order (e.g., CW, CCW). This would be consistent with the preponderance of Neg/Pos Rebounding that we observed in Experiment 2 following rebounding primes. A second possibility is that rebounding motion itself can be primed by a high-level mechanism that (1) encodes a sequence of motion steps (i.e., two frame transitions), (2) maintains their temporal order during the random frames, and (3) deploys selective attention during each random frame transition, reinstantiating the encoded motion pattern. This type of priming could operate regardless of the underlying inhibitory and excitatory relationships between directionally selective neural populations. Although we cannot distinguish between these two possibilities on the basis of our data, a future study could address this question by examining whether it is possible to prime two-step motion patterns that involve nonopposing directions (e.g., "staircase" motion that proceeds up-right-up-right . . . ) or even longer multistep motion patterns (e.g., up-up-down-down ....). If it is possible to prime such nonrebounding multistep motion patterns, this would be evidence of a higher-order, memory-based mechanism.

\section{Program and data availability}

The experiment and analysis scripts as well as the raw data files can be accessed at https://osf.io/9ct5n/.

\section{References}

Anstis, S., \& Ramachandran, V. S. (1987). Visual inertia in apparent motion. Vision Research, 27, 755-764.

Culham, J. C., Verstraten, F. A., Ashida, H., \& Cavanagh, P. (2000). Independent aftereffects of attention and motion. Neuron, 28, 607615.

Davidenko, N., Cheong, Y., \& Smith, J. (2015). The suggestible nature of apparent motion perception. In D. C. Noelle, R. Dale, A. S. Warlaumont, J. Yoshimi, T. Matlock, C. D. Jennings, \& P. P. Maglio (Eds.), Proceedings of the 37th Annual Meeting of the Cognitive Science Society (pp. 513-517). Austin, TX: Cognitive Science Society.

Davidenko, N., Heller, N. H., Cheong, Y., \& Smith, J. (2017). Persistent illusory apparent motion in sequences of uncorrelated random dots. Journal of Vision, 17(3), 19. https://doi.org/10.1167/17.30.19

Heller, N. H., \& Davidenko, N. (2018). Dissociating higher and lower order visual motion systems by priming illusory apparent motion. Perception, 47, 30-43. https://doi.org/10.1177/0301006617731007

Hock, H. S., Park, C. L., \& Schöner, G. (2002). Self-organized pattern formation: Experimental dissection of motion detection and motion integration by variation of attentional spread. Vision Research, 42, 991-1003.

Hsieh, P. J., Caplovitz, G. P., \& Tse, P. U. (2005). Illusory rebound motion and the motion continuity heuristic. Vision Research, 45, 2972 2985.

Hsieh, P. J., \& Tse, P. U. (2006). Stimulus factors affecting illusory rebound motion. Vision Research, 46, 1924-1933.

Kanai, R., \& Verstraten, F. A. (2005). Perceptual manifestations of fast neural plasticity: Motion priming, rapid motion aftereffect and perceptual sensitization. Vision Research, 45, 3109-3116.

Laing, C. R., \& Chow, C. C. (2002). A spiking neuron model for binocular rivalry. Journal of Computational Neuroscience, 12, 39-53.

Lehky, S. R. (1988). An astable multivibrator model of binocular rivalry. Perception, 17, 215-228.

Maloney, L. T., Dal Martello, M. F., Sahm, C., \& Spillmann, L. (2005). Past trials influence perception of ambiguous motion quartets through pattern completion. Proceedings of the National Academy of Sciences, 102, 3164-3169.

Mueller, T. J. (1990). A physiological model of binocular rivalry. Visual Neuroscience, 4, 63-73.

Nishida, S. Y., \& Sato, T. (1992). Positive motion after-effect induced by bandpass-filtered random-dot kinematograms. Vision Research, 32, 1635-1646.

Nishida, S. Y., \& Sato, T. (1995). Motion aftereffect with flickering test patterns reveals higher stages of motion processing. Vision Research, 35, 477-490.

Ramachandran, V. S., \& Anstis, S. M. (1985). Perceptual organization in multistable apparent motion. Perception, 14, 135-143.

Ramachandran, V. S., \& Cavanagh, P. (1987). Motion capture anisotropy. Vision Research, 27, 97-106. 
Takeuchi, T., Tuladhar, A., \& Yoshimoto, S. (2011). The effect of retinal illuminance on visual motion priming. Vision Research, 51, 11371145.

Verstraten, F. A. J., Cavanagh, P., \& Labianca, A. T. (2000). Limits of attentive tracking reveal temporal properties of attention. Vision
Research, 40, 3651-3664. https://doi.org/10.1016/S0042-6989(00) 00213-3

Yoshimoto, S., Uchida-Ota, M., \& Takeuchi, T. (2014). The reference frame of visual motion priming depends on underlying motion mechanisms. Journal of Vision, 14(1), 10. https://doi.org/10.1167/14.1.10 